\title{
HIV and HIV dementia
}

Departments of Neurology and Microbiology, University of Pennsylvania School of Medicine, Philadelphia, Pennsylvania, USA

Address correspondence to: Francisco González-Scarano, Department of Neurology, University of Pennsylvania, 3 West Gates, Philadelphia, Pennsylvania 19104-4283, USA. Phone: (215) 662-3360; Fax: (215) 662-3362;

E-mail:SCARANO@mail.med.upenn.edu.

Lentiviruses, a group of retroviruses that includes HIV-1, have been known to be neurovirulent since landmark studies by Sigurdsson and others, who showed that chronic lentiviral infections could take hold in the central nervous system (CNS; reviewed in ref. 1). Since their discovery, HIV-1 and, to a lesser extent, HIV-2 have been recognized as CNS pathogens that can cause a subcortical dementia (HIV-associated dementia; HAD) in a significant proportion of infected patients (2). In $\mathrm{HAD}$, as distinct from conventional cortical dementias, memory impairment typically occurs either in association with or following other cognitive symptoms like inattention, apathy, and psychomotor slowing. Motor symptoms such as lack of coordination are also characteristic of this condition.

The neuropathological changes induced by HIV- 1 infection of the CNS include pallor of the myelin sheaths, proliferation or prominence of astrocytes, and the formation of multinucleated giant cells (syncytia). Microglia and brain macrophages are the main cellular targets for HIV infection in the CNS, and syncytium formation is a signature finding for this infection, since it results from interactions between the viral glycoproteins gp120 and gp41 and the principal cellular HIV-1 receptors in microglia, CD4 and CCR5. The presence of multinucleated giant cells in the CNS is the most specific finding in HIV infection, and it is roughly correlated with the antemortem diagnosis of HAD (3).

\section{Microglia and neurological damage in HAD}

Most strikingly, the neurons of patients with HAD do not appear to be infected with HIV-1, although commonly there is other evidence of neuronal pathology, including neuronal dropout, abnormalities of dendritic processes, and apoptotic cell death (4-7). Neuronal abnormalities in $\mathrm{HAD}$, whether strictly physiological or accompanied by morphological changes, appear to be the indirect result of microglial infection, perhaps mediated by the release of soluble toxic factors from HIV-1 infected microglia (reviewed in ref. 8). Among these putative toxins are the viral proteins gp120, gp41, and tat, the viral transactivator, or alternatively, macrophage products such as TNF- $\alpha$, glutamate, or quino-

Since many antiretroviral agents do not penetrate the brain, and since microglia are exceedingly long-lived, it is possible that these cells serve as a reservoir in which HIV-1 persists even after systemic eradication.

Unfortunately, viral load in the brains of people with HIV-associated dementia cannot be assayed directly but must be inferred from indirect measures.

linic acid. The effects of gp120 on neurons have received increasing attention since the recent demonstration that many neuronal populations express the chemokine receptors that, together with CD4, are responsible for viral binding to cell membranes (9-16).

Besides the scientifically interesting questions associated with unraveling the mechanism of HAD, there are pressing issues regarding the critical role of virus replication within the brain in mediating this complication (17-22). Since many antiretroviral agents do not penetrate the brain, and since microglia are exceedingly long- lived, it is possible that these cells serve as a reservoir in which HIV-1 persists even after systemic eradication. Unfortunately, viral load in HAD cannot be measured directly but must be inferred from neuroimaging data or from assays of the cerebrospinal fluid, which may not reflect the situation in the brain parenchyma. Hence, the class of data that has permitted immunodeficiency to be associated in a convincing manner with HIV plasma and lymphoid tissue RNA levels cannot be obtained for the CNS. However, these indirect methods have suggested that aggressive treatment with highly active antiretroviral therapy (HAART) reverses certain magnetic resonance spectroscopic changes that are characteristic of HIV infection of the brain (23), but also that the dynamics of viral clearance from the cerebrospinal fluid (CSF) have unique kinetics (17). Thus, although HAART appears to have an effect on the clinical and laboratory parameters that indicate brain disease, the brain may nevertheless require special attention when pharmacotherapeutic regimens are designed.

\section{Simian immunodeficiency virus and neurological dysfunction}

Because the simian immunodeficiency virus (SIV) macaque model parallels HIV infection in its brain pathology, as well as in its induction of psychomotor slowing, this system may provide a means to study the importance of viral levels in the generation of CNS abnormalities. One previous study clearly showed that the pathological changes of SIV infection of the brain are correlated with virus levels (24). The current study by Fox et al. in this issue of the JCI (25) goes one step further, analyzing the effect of antiretroviral treatment on an important physiological parameter - brainstem auditory evoked responses. 
Fox et al. inoculated four rhesus macaques intravenously with a macrophage-adapted virus (SIVmac182) and then assessed the animals longitudinally by quantifying brainstem auditory-evoked potentials (BSAEPs), motor movements, body temperature, and viral load (25). BSAEPs are performed by presenting an auditory stimulus (click) to one ear, and then recording electrical waveforms through surface electrodes. Typically five sequential waveforms are generated within structures in the auditory nerve and brainstem. BSAEPs provide an objective measure of electrical conduction latencies of neuronal pathways leading from auditory input regions of the lower pons rostrally through the midbrain. Such testing, which is extraordinarily sensitive to regional disturbances in neuronal cell function throughout these anatomic sites, can localize either destructive or reversible lesions within these conduction pathways. Within 1 month of inoculation, the BSAEPs became abnormal, indicated by conduction disturbance in the waveform generated within the upper brainstem/midbrain region. Antiviral therapy with 9-(2-phosphonylmethoxypropyl)adenine (PMPA) resulted in rapid reduction of plasma viral load and, subsequently, in restoration of more normal BSAEPs, demonstrating that the early neurological defects in this system are reversible. Discontinuing therapy resulted in a rapid viral rebound. This was followed by reappearance of the same conduction delay, consistent with a cause-effect relationship between high viral load and abnormal neurophysiology.

While these brainstem conduction abnormalities were clearly reversible, the motor disturbances and baseline body temperature elevations were not. The failure to reverse gross motor deficits with antiretroviral therapy suggests permanent tissue destruction in acutely infected animals; however, neither the severity of histopathological damage nor the level of virus expression within brain parenchyma or CSF correlated with the severity of the conduction abnormalities.

\section{Relevance to human neurological disease}

The early brain damage seen in this model is surprising in view of our understanding of the evolution of chronic neurodegeneration in HAD, in which the damage occurs later in the course of the infection. Nonetheless, the normalization of BSAEPs in treated animals is consistent with the observed improvement in neuropsychological test performance seen in adult and pediatric patients treated with antiretrovirals, particularly zidovudine $(26,27)$. Although the reversible conduction signal abnormalities in macaques are clearly generated within the brainstem, it is unclear how PMPA treatment influences them, since there is no evidence that this drug enters either the brain parenchymal or CSF compartments. Whether the improvement is a secondary effect of the suppression of peripheral viral replication, the suppression of replication in immuneactivated trafficking cells, the suppression of soluble factors entering the brain parenchyma from immuneactivated cells in the vascular space or within brain parenchyma, or other effects is open to speculation.

The number of animals examined in the work of Fox et al. (25) was too small to allow conclusions concerning the utility of CSF viral load in predicting neurological dysfunction, but the sensitivity and ease of administration of the BSAEPs suggest that they may be a clinically useful tool for assessing patients at risk for neurological dysfunction. Such noninvasive, quantitative paraclinical tests are clearly critical for understanding CNS damage in AIDS and for assessing responses to current and future therapies. Currently, neuroradiology is providing quantitative functional and structural assessment of brain integrity in HIV infection $(23,28)$, and Fox et al.'s study (25) adds supportive and independent evidence of early functional brain disturbances in SIV infection. While it is heartening that some of the damage induced by SIV is reversible, other early changes are clearly irreversible. Moreover, the finding that interruption of therapy permitted abnormal BSAEPs to return is disappointing for several reasons, notably because it suggests that the virus can persist within the CNS. If these results can be extrapolated to humans, then it is likely that "drug holidays" would allow a return of CNS abnormalities, much as they result in rebounds in plasma viremia. Thus, these experiments predict that the CNS will be a reservoir for chronic persistence of HIV and that continual viral suppression in both the CNS and plasma will be necessary to protect the brain.

1. Nathanson, N. 1998. Neurologic disease produced by visna maedi and caprine arthritisencephalitis viruses, lentiviruses of sheep and goats. In The neurology of AIDS. H.E. Gendelman, S.A. Lipton, L. Epstein, and S. Swindells, editors. Chapman \& Hall. New York, New York, USA. 179-188.

2. McArthur, J.C., and Grant, I. 1998. HIV neurocognitive disorders. In The neurology of AIDS H.E. Gendelman, S.A. Lipton, L. Epstein, and S. Swindells, editors. Chapman \& Hall. New York, New York, USA. 499-523.

3. Glass, J.D., Fedor, H., Wesselingh, S.L., and McArthur, J.C. 1995. Immunocytochemical quantitation of human immunodeficiency virus in the brain: correlations with dementia. Ann. Neurol. 38:755-762.

4. Masliah, E., et al. 1997. Dendritic injury is a pathological substrate for human immunodeficiency virus-related cognitive disorders. Ann. Neurol. 42:963-972.

5. Gelbard, H.A., et al. 1995. Apoptotic neurons in brains from paediatric patients with HIV-1 encephalitis and progressive encephalopathy. Neuropathol. Appl. Neurobiol. 21:208-217.

6. Adle-Biassette, H., et al. 1995. Neuronal apoptosis in HIV infection in adults. Neuropathol. Appl. Neurobiol. 21:218-227.

7. Petito, C.K., and Roberts, B. 1995. Evidence of apoptotic cell death in HIV encephalitis. Am. J. Pathol. 146:1121-1130.

8. Gendelman, H.E., et al. 1997. The neuropathogenesis of the AIDS dementia complex. AIDS. 11(Suppl. A):S35-S45.

9. Lavi, E., et al. 1997. CXCR-4 (Fusin), a co-receptor for the type 1 human immunodeficiency virus (HIV-1), is expressed in the human brain in a variety of cell types, including microglia and neurons. Am. J. Pathol. 151:1035-1042.

10. Hesselgesser, J., et al. 1997. CD4-independent association between HIV-1 gp120 and CXCR4: functional chemokine receptors are expressed in human neurons. Curr. Biol. 7:112-121.

11. Meucci, O., et al. 1998. Chemokines regulate hippocampal neuronal signaling and gp120 neurotoxicity. Proc. Natl. Acad. Sci. USA. 95:14500-14505.

12. Kaul, M., and Lipton, S.A. 1999. Chemokines and activated macrophages in HIV gp120-induced neuronal apoptosis. Proc. Natl. Acad. Sci. USA. 96:8212-8216.

13. Westmoreland, S.V., Rottman, J.B., Williams, K.C., Lackner, A.A., and Sasseville, V.G. 1998 Chemokine receptor expression on resident and inflammatory cells in the brain of macaques with simian immunodeficiency virus encephalitis. Am. J. Pathol. 152:659-665.

14. Vallat, A.V., et al. 1998. Localization of HIV-1 coreceptors CCR5 and CXCR4 in the brain of children with AIDS. Am. J. Pathol. 152:167-178.

15. Sasseville, V.G., et al. 1996. Chemokine expres- 
sion in simian immunodeficiency virus-induced AIDS encephalitis. Am. J. Pathol. 149:1459-1467.

16. Coughlan, C.M., et al. 2000. Expression of multiple functional chemokine receptors and MCP-1 chemokine in human neurons. Neuroscience. 97:591-600.

17. Ellis, R.J., et al. 2000. Cerebrospinal fluid HIV RNA originates from both local CNS and systemic sources. Neurology. 54:927-936.

18. Tozzi, V., et al. 1999. Positive and sustained effects of highly active antiretroviral therapy on HIV-1-associated neurocognitive impairment. AIDS. 13:1889-1897.

19. Brew, B.J., Pemberton, L., Cunningham, P., and Law, M.G. 1997. Levels of human immunodeficiency virus type 1 RNA in cerebrospinal fluid correlate with AIDS dementia stage. J. Infect. Dis.
175:963-966.

20. McArthur, J.C., et al. 1997. Relationship between human immunodeficiency virus-associated dementia and viral load in cerebrospinal fluid and brain. Ann. Neurol. 42:689-698.

21. Childs, E.A., et al. 1999. Plasma viral load and CD4 lymphocytes predict HIV-associated dementia and sensory neuropathy. Neurology. 52:607-613.

22. Dore, G.J., et al. 1999. Changes to AIDS dementia complex in the era of highly active antiretroviral therapy. AIDS. 13:1249-1253.

23. Chang, L., et al. 1999. Highly active antiretroviral therapy reverses brain metabolite abnormalities in mild HIV dementia. Neurology. 53:782-789.

24. Zink, M.C., et al. 1999. High viral load in the cerebrospinal fluid and brain correlates with severity of simian immunodeficiency virus encephalitis. $J$.
Virol. 73:10480-10488.

25. Fox, H.S., et al. 2000. Antiviral treatment normalizes central nervous system-evoked potential but not movement abnormalities in simian immunodeficiency virus-infected monkeys. J. Clin. Invest. 106:37-45.

26. Pizzo, P.A., et al. 1988. Effect of continuous intravenous infusion of zidovudine (AZT) in children with symptomatic HIV infection. N. Engl. J. Med. 319:889-896.

27. Schmitt, F.A., et al. 1988. Neuropsychological outcome of zidovudine (AZT) treatment of patients with AIDS and AIDS-related complex. $N$ Engl. J. Med. 319:1573-1578.

28. Yiannoutsos, C., et al. 2000. Patterns of regional brain metabolism in AIDS dementia complex. Neurology. 54:A251. (Abstr.) 